\title{
Change in the diagnosis of inflammatory bowel disease: a hospital-based cohort study from Korea
}

\author{
Ho-Su Lee ${ }^{1}$, Jaewon Choe ${ }^{1}$, Hyo Jeong Lee ${ }^{1}$, Sung Wook Hwang ${ }^{2}$, Sang Hyoung Park ${ }^{2}$, Dong-Hoon Yang ${ }^{2}$, \\ Kyung-Jo Kim², Byong Duk Ye², Jeong-Sik Byeon'², Seung-Jae Myung ${ }^{2}$, Yong Sik Yoon ${ }^{3}$, Chang Sik Yu ${ }^{3}$, \\ Jin-Ho Kim², Suk-Kyun Yang ${ }^{2}$ \\ ${ }^{1}$ Health Screening and Promotion Center, ${ }^{2}$ Department of Gastroenterology, ${ }^{3}$ Colon and Rectal Surgery, University of Ulsan College of Medicine, \\ Asan Medical Center, Seoul, Korea
}

\begin{abstract}
Background/Aims: Accurately diagnosing inflammatory bowel disease (IBD) remains a challenge, but is crucial for providing proper management for affected patients. The aim of the present study was to evaluate the frequency of change in diagnosis in Korean patients who were referred to our institution with a diagnosis of IBD. Methods: We enrolled 1,444 patients diagnosed with ulcerative colitis (UC) and 1,452 diagnosed with Crohn's disease (CD), who had been referred to the Asan Medical Center between January 2010 and December 2014. These patients were assessed and subsequently classified as having UC, CD, indeterminate colitis, possible IBD, or non-IBD. Results: During a median follow-up of 15.9 months, 400 of the 2,896 patients (13.8\%) analyzed in this study experienced a change in diagnosis. A change in diagnosis from UC to CD, or vice-versa, was made in 24 of 1,444 patients (1.7\%) and 23 of 1,452 patients (1.6\%), respectively. A change to a non-IBD diagnosis was the most common modification; 7.5\% (108 of 1444) and 12.7\% (184 of 1452) of the patients with a referral diagnosis of UC and CD, respectively, were reclassified as having non-IBD. Among the 292 patients who were ultimately determined not to have IBD, 135 (55 UC and 80 CD cases) had received IBD-related medication. Conclusions: There are diagnostic uncertainties and difficulties in relation to IBD. Therefore, precise assessment and systematic follow-up are essential in the management of this condition. (Intest Res 2016;14:258-263)
\end{abstract}

Key Words: Crohn disease; Ulcerative colitis; Diagnosis; Inflammatory bowel diseases

\section{INTRODUCTION}

The exact diagnosis of IBD is crucial for patient safety and treatment and for the interpretation of epidemiological data. ${ }^{1-3}$ However, diagnosis and sub-classification of IBD are sometimes not obvious even after clinical, endoscopic,

Received March 23, 2016. Revised May 2, 2016.

Accepted May 9, 2016.

Correspondence to Suk-Kyun Yang, Department of Gastroenterology, University of Ulsan College of Medicine, Asan Medical Center, 88 Olympic-ro

43-gil, Songpa-gu, Seoul 05505, Korea. Tel: +82-2-3010-3901, Fax: +82-2-

476-0824,E-mail:sky@amc.seoul.kr

Financial support: This work was supported by a grant from the Korean Health Technology R\&D Project, Ministry of Health \& Welfare, Republic of Korea [A120176]. Conflict of interest: Suk-Kyun Yang received a research grant from Janssen Korea Ltd. but this grant is not related to the topic of the current study. The remaining authors have no conflicts of interest to declare. radiologic, macroscopic, and histologic evaluations. ${ }^{4,5}$ In practice, $3 \%-10 \%$ of patients with colonic inflammation have overlapping clinical and pathological features, making it difficult to distinguish between UC and CD, and resulting in the diagnosis of indeterminate colitis (IC).$^{6-8}$ Furthermore, a change in diagnosis was reported after reevaluation during follow-up in $10 \%$ of patients originally diagnosed with UC or CD in the Inflammatory Bowel South-Eastern Norway (IBSEN) cohort. ${ }^{1,3,9,10}$ This finding highlights not only the heterogeneity of these diseases, but also the difficulty of ascertaining their correct diagnoses.

The incidence of IBD has been rapidly increasing in Asian countries, and physicians have become more aware of and more knowledgeable about this condition. ${ }^{11,12}$ However, to the best of our knowledge, no studies to date have systemati-

\footnotetext{
๑ Copyright 2016. Korean Association for the Study of Intestinal Diseases. All rights reserved.

This is an Open Access article distributed under the terms of the Creative Commons Attribution Non-Commercial License (http://creativecommons.org/licenses/by-nc/4.0)

which permits unrestricted non-commercial use, distribution, and reproduction in any medium, provided the original work is properly cited.
} 
cally evaluated the difficulties and pitfalls in diagnosing IBD with its rapid shifts in incidence in this region. The aim of the present study was to address this issue by evaluating the frequency with which alterations in the diagnosis of IBD occur in Korea, and to create a list of diseases mimicking this disorder.

\section{METHODS}

\section{Patients}

The medical records of 2,896 patients who were referred to Asan Medical Center with a diagnosis of IBD between January 2010 and December 2014 were collected from the Asan IBD registry. ${ }^{13,14}$ These patients were referred from private clinics, local hospitals, and university hospitals.

\section{Study Design}

Data regarding clinical information, including radiologic imaging, endoscopy, and histological reports from the referring physicians were reviewed. The diagnoses originally provided by the referring physicians or patients were either changed or confirmed according to assessments by eight expert gastroenterologists (H.S.L., S.H.P., D.H.Y., K.J.K., B.D.Y., J.S.B., S.J.M., and S.K.Y.). The patients were subsequently reclassified as having UC, CD, IC, possible IBD, or non-IBD. To minimize misjudgment, a diagnosis was changed only when two or more reviewers agreed, and any discrepancies regarding reclassification were resolved by consensus. The diagnosis of CD and UC was determined based on conventional clinical, radiologic, endoscopic, and histopathologic criteria. ${ }^{12,15-19}$ The diagnostic criteria for the classification of IC were clear evidence of IBD and divergent or inconclusive endoscopy and histopathology results for a diagnosis of definite UC or CD. ${ }^{8}$ The term "possible IBD" was used in cases where the quality of the diagnostic examination was not satisfactory or the findings were ambiguous. ${ }^{1,3}$ The most probable final diagnosis and the time lag from the initial diagnosis of IBD to reclassification of the diagnosis as nonIBD were investigated for each patient who was ultimately deemed not to have IBD (non-IBD). The follow-up duration was calculated from the day of the first visit to our center to the last follow-up visit.

\section{Statistical Analysis}

Continuous variables were expressed as medians with ranges; discrete data were tabulated as numbers and percentages. Statistical evaluations were performed using the statistical software package SPSS 21.0 for Windows (IBM SPSS, ver. 21.0; IBM Corp., Armonk, NY, USA). The institutional review board of Asan Medical Center approved this retrospective study (IRB No. 2016-0021).

\section{RESULTS}

\section{Baseline Characteristics}

The baseline characteristics of the patients are listed in Table 1. Of the 2,896 patients included in the current study, $57.4 \%(829$ of 1,444$)$ and $70.0 \%(1,017$ of 1,452$)$ were men with alleged UC and CD, respectively. The median age at ini-

Table 1. Baseline Characteristics of the Study Patients

\begin{tabular}{|c|c|c|}
\hline Variable & Referred as UC $(n=1,444)$ & Referred as CD $(n=1,452)$ \\
\hline Age at initial diagnosis, yr & $36(9-80)$ & $22(9-77)$ \\
\hline Median interval from diagnosis to referral, mo & $11.0(0.1-1380.3)$ & $5.8(0.1-1381.1)$ \\
\hline \multicolumn{3}{|l|}{ Institution of first diagnosis } \\
\hline Private clinics & 471 (32.6) & 217 (14.9) \\
\hline University hospitals & $387(26.8)$ & $722(49.7)$ \\
\hline \multicolumn{3}{|l|}{ Institution of referral } \\
\hline Private clinics & $371(25.7)$ & $182(12.5)$ \\
\hline Local hospitals & $551(38.2)$ & $472(32.5)$ \\
\hline
\end{tabular}

Values are presented as $n(\%)$ or $n$ (range). 
tial diagnosis was 36 years (range: 9-80 years) in the cases of alleged UC and 22 years (range: 9-77 years) in the cases of alleged CD.
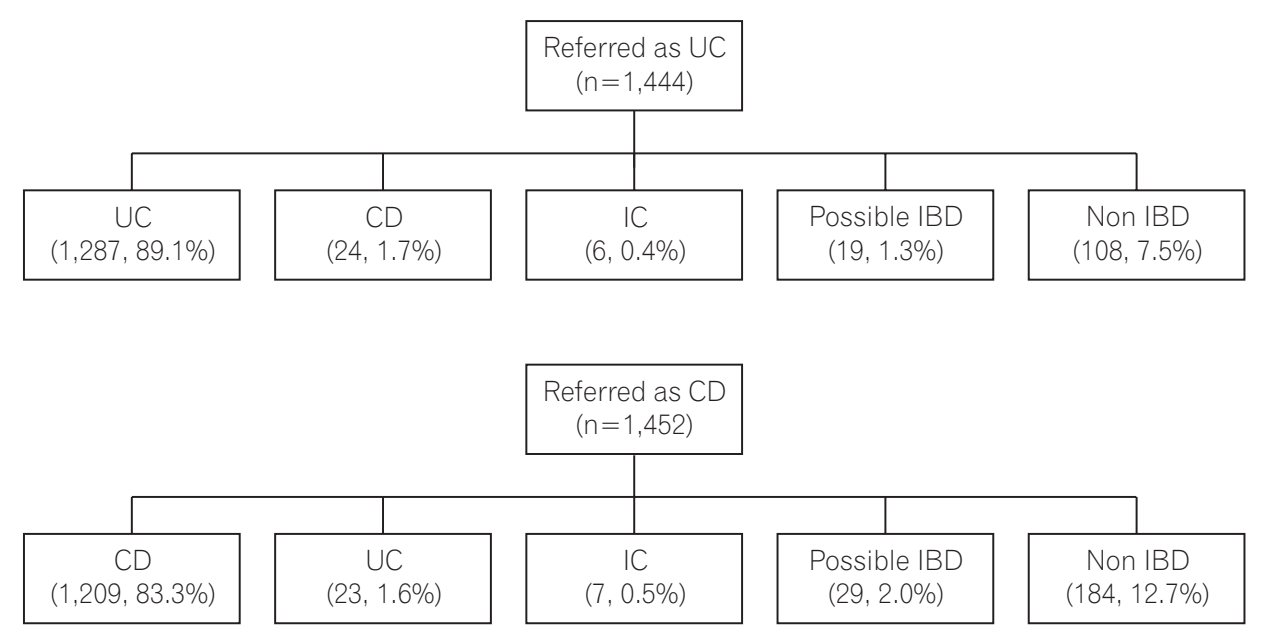

Fig. 1. Final diagnosis according to the initial diagnosis. Patients who were referred with the diagnosis of UC or CD were subsequently classified as having $U C_{1} C D_{\text {, }}$ indeterminate colitis $(\mathrm{IC})$, possible IBD, or non-IBD.

Table 2. Diseases that Mimicked UC and CD in the Study Population

\begin{tabular}{llll}
\hline \multicolumn{1}{c}{ Misdiagnosed as UC } & $\mathbf{n = 1 0 8}$ & \multicolumn{1}{c}{ Misdiagnosed as CD } & $\mathbf{n = 1 8 4}$ \\
\hline Acute self-limited colitis & $66(61.1)$ & Acute self-limited colitis & $57(31.0)$ \\
Intestinal tuberculosis & $6(5.6)$ & Intestinal tuberculosis & $53(28.8)$ \\
Mucosal prolapse syndrome & $6(5.6)$ & Isolated terminal ileal ulcerations & $18(9.8)$ \\
\hline Pseudomembranous colitis & $6(5.6)$ & Behçet's disease & $14(7.6)$ \\
Drug-induced colitis & $5(4.6)$ & Lymphoid hyperplasia of terminal ileum & $6(3.3)$ \\
Ischemic colitis & $5(4.6)$ & Drug-induced colitis & $5(2.7)$ \\
Lymphoid hyperplasia of rectum & $3(2.8)$ & Cancer & $5(2.7)$ \\
Cap polyposis & $2(1.9)$ & Amebic colitis & $4(2.2)$ \\
Eosinophilic colitis & $2(1.9)$ & Eosinophilic colitis & $3(1.6)$ \\
Radiation colitis & $1(0.9)$ & Appendicitis & $3(1.6)$ \\
Diversion colitis & $1(0.9)$ & Diverticulitis & $2(1.1)$ \\
Diabetes-associated diarrhea & $1(0.9)$ & Pseudo-obstruction & $2(1.1)$ \\
Amebic colitis & $1(0.9)$ & Cryptogenic multifocal ulcerous stenosing enteritis & $2(1.1)$ \\
Polyposis syndrome & $1(0.9)$ & Bile salt diarrhea & $1(0.5)$ \\
Systemic lupus erythematosus-associated colitis & $1(0.9)$ & Cytomegalovirus colitis & $1(0.5)$ \\
Behçet's disease & $1(0.9)$ & Radiation colitis & $1(0.5)$ \\
& & Ischemic colitis & $1(0.5)$ \\
& & Small intestinal bacterial overgrowth & $1(0.5)$ \\
& & Henoch-Schönlein purpura & $1(0.5)$ \\
\hline
\end{tabular}

Values are presented as $n(\%)$ 


\section{Change in the Diagnosis of IBD}

Over a median of 15.9 months of follow-up, 400 of 2,896 patients (13.8\%) diagnosed with IBD experienced a change in the original diagnosis. Of the patients initially diagnosed with UC, $89.1 \%(1,287$ of 1,444$)$ had their diagnosis confirmed, compared with $83.3 \%(1,209$ of 1,452$)$ of patients initially diagnosed with CD (Fig. 1). The referral diagnosis of UC was confirmed in 82.2\% (305 of 371), 89.5\% (493 of 551), and $93.7 \%$ (489 of 522 ) of patients referred from primary clinics, local hospitals, and university hospitals, respectively. Likewise, the referral diagnosis of CD was confirmed in $65.9 \%$ ( 120 of 182), 82.2\% (388 of 472), and $87.8 \%$ (701 of 798) of patients referred from primary clinics, local hospitals, and university hospitals, respectively. In the majority of cases with amendments, the diagnosis was changed from UC or CD to non-IBD (diagnosis changed from UC to nonIBD: 7.5\%; diagnosis changed from CD to non-IBD: 12.7\%). A relatively small proportion of patients originally diagnosed with UC or CD required their diagnosis to be changed to another form of IBD (from UC to CD: 1.7\%; from CD to UC: $1.6 \%)$. Thirteen patients $(0.4 \%)$ were reclassified as having IC, and 48 patients (1.7\%) as having possible IBD.

\section{The Mimics of IBD}

The distribution of IBD mimics is presented in Table 2. In the cases of alleged UC, acute self-limited colitis ( $\mathrm{n}=66$ ) was the main mimic identified, followed by intestinal tuberculosis (ITB) $(n=6)$, mucosal prolapse syndrome $(n=6)$, pseudomembranous colitis $(n=6)$, drug-induced colitis $(n=5)$, and ischemic colitis $(n=5)$. In the case of alleged CD, the most common mimic was acute self-limited colitis $(n=57)$. Other common mimics included ITB $(\mathrm{n}=53)$, isolated terminal ileal ulcerations ( $\mathrm{n}=18)$, Behçet's disease $(\mathrm{n}=14)$, lymphoid hyperplasia of the terminal ileum ( $\mathrm{n}=6$ ), drug-induced colitis $(\mathrm{n}=5)$, and cancer $(\mathrm{n}=5)$.

\section{Reclassification as Non-IBD}

The median time lag from the initial diagnosis of IBD to a reclassification as non-IBD was 2.0 months (range: 0.0-92.4 months) for UC and 3.3 months (range: 0.2-132.1 months) for CD patients. Among the 292 patients ultimately deemed not to have IBD, 52 cases ( 14 of 108 patients diagnosed with UC and 38 of 184 patients diagnosed with CD) had been suspected of having IBD for more than 1 year. IBD-related medications (namely, 5-aminosalicylates, thiopurines, corti-
Table 3. IBD-related Medications Used in the Patients Ultimately Deemed not to have IBD

\begin{tabular}{lcc}
\hline \multicolumn{1}{c}{ Variable } & $\begin{array}{c}\text { Misdiagnosed } \\
\text { as UC } \\
(\mathbf{n = 1 0 8})\end{array}$ & $\begin{array}{c}\text { Misdiagnosed } \\
\text { as CD } \\
(\mathbf{n = 1 8 4})\end{array}$ \\
\hline None & $53(49.1)$ & $104(56.5)$ \\
5-aminosalicylates & $52(48.1)$ & $75(40.8)$ \\
Thiopurines & $1(0.9)$ & $11(6.0)$ \\
Corticosteroids & $15(13.9)$ & $28(15.2)$ \\
Anti-tumor necrosis factor agents & $1(0.9)$ & 0 \\
\hline
\end{tabular}

Values are presented as $n(\%)$.

costeroids, and anti-tumor necrosis factor [TNF]) were used in 55 of the 108 patients (50.9\%) misdiagnosed with UC and in 80 of the 184 patients (43.5\%) misdiagnosed with CD (Table 3). We also evaluated the time lag from the first visit to the Asan Medical Center to reclassification as non-IBD. The change in diagnosis from IBD to non-IBD was made at the first visit or within 2 months after the first visit to the Asan Medical Center in 267 of 292 patients (91.4\%). In the remaining 25 patients (8.6\%), the change in diagnosis was made after a follow-up period of between 2 months and 36 months.

\section{DISCUSSION}

We show from our current analysis that a change in diagnosis after a careful review and evaluation was required for 400 of 2,896 patients (13.8\%) initially alleged to have IBD. The main change in the diagnosis of patients who were referred with a diagnosis of IBD was a reclassification as non-IBD.

An exact diagnosis of IBD is essential for its proper management. However, there are several hurdles when diagnosing and classifying this condition. Inflammatory changes in the bowel observed by radiology and endoscopy are frequently non-characteristic. Therefore, inflammatory changes in the bowel such as an infectious condition or ischemic colitis cannot be immediately or easily distinguished from IBD. ${ }^{4,5,20}$ Second, the dynamic nature of IBD often makes it complex to differentiate between patients with IBD who achieve a complete remission after a short period of first attack and patients with self-limiting colitis. ${ }^{9,10,21}$ Moreover, the overlap between CD and UC in terms of clinical, endoscopic, and pathological findings could make distinguishing between these two diseases difficult, particularly when the lesions are subtle or fulminant. ${ }^{8}$ However, these conditions become more discernible with follow-up; thus, patients with suspected IBD need to be evaluated through observation 
over time.

Consistent with previous studies from IBSEN ${ }^{1,3} 13.8 \%$ of the patients in our current series who were initially diagnosed with IBD had a change in diagnosis, mainly a reclassification to non-IBD. The most frequent cause of misclassification in patients misdiagnosed with either UC or CD was acute self-limited colitis. A systematic follow-up should lead to reductions in such misdiagnoses. ITB also remains a major hurdle in the accurate diagnosis of IBD in Korea. ${ }^{12,22-25}$ Although previous studies have suggested and evaluated diagnostic tools, ${ }^{22,24,26-29}$ the differential diagnosis of CD and ITB remains challenging, particularly in areas with a high prevalence of ITB. ${ }^{28,29}$ Owing to the rarity of CD among Koreans, a significant proportion of Korean patients with CD have been misdiagnosed with ITB. However, with the rising incidence and prevalence of $\mathrm{CD}$ and with the increasing awareness of CD among physicians in Korea, ${ }^{11,12,30}$ ITB cases initially misdiagnosed as CD are increasing. Therefore, physicians should be more cautious while evaluating the differential diagnosis because the diagnostic confusion between CD and ITB may result in the inappropriate use or even misuse of medications that could be harmful to the patient.

The accuracy of the diagnosis and sub-classification of IBD could also have a significant impact on the results of clinical studies. In hospital-based studies, a diagnosis is usually reassessed because the patient's clinical course has been followed over time. However, in epidemiology studies using administrative databases, the information in the databases has the inherent challenge of misclassification bias even by using validated codes. ${ }^{31-33}$ An administrative database cannot capture detailed and revised medical records, and thus, precise approaches in designing (particularly patient selection), analyzing, and carefully interpreting the results are necessary. Moreover, confounding by misdiagnosis and misclassification is critically relevant in genetic studies. ${ }^{2,34}$ Making an accurate diagnosis and constantly updating clinical medical records are the principles for analyzing and interpreting genetic data.

Clinically relevant findings from our current analyses include the fact that $46.2 \%$ of the patients (135 of 292) analyzed received an IBD-related medication during a misdiagnosis. Furthermore, over 10\% of misdiagnosed patients received thiopurines, corticosteroids, or anti-TNF agents. These medications could have significant adverse effects, especially in patients with infections. ${ }^{35}$ Above all, the misdiagnosis of ITB as IBD can lead to serious consequences because immunosuppressive drugs, such as corticosteroids, thiopurines, and anti-TNF agents can actually aggravate ITB.
Thus, patients with a questionable diagnosis of IBD should be carefully observed at follow-up, and caution should be exercised by physicians during the administration of any medications.

Our study had a few limitations of note. First, our analysis was retrospective in nature. Therefore, the diagnostic tools and work-ups were not controlled, and a pathologic evaluation was not performed on all patients. Second, there was a chance that we still incorrectly diagnosed some of our patients. However, the diagnosis was only deemed conclusive when two or more gastroenterologists independently classified a case identically, and any discrepancies were resolved by discussion. Third, the follow-up period may not have been sufficient. Confidence in a diagnosis has been shown to increase with a longer duration of follow-up. Thus, future studies with a longer follow-up are necessary to determine the risk of IBD misdiagnosis.

In conclusion, numerous diseases can mimic IBD clinically, but careful and repeated evaluations reduce the chance of the misdiagnosis and misclassification of this disorder. Careful assessment of patients, systematic follow-up, and continuous correction or updating of the information in medical records will facilitate correct diagnoses and a reduction in misclassification bias.

\section{REFERENCES}

1. Moum B, Ekbom A, Vatn MH, et al. Inflammatory bowel disease: re-evaluation of the diagnosis in a prospective population based study in south eastern Norway. Gut 1997;40:328-332.

2. Silverberg MS, Daly MJ, Moskovitz DN, et al. Diagnostic misclassification reduces the ability to detect linkage in inflammatory bowel disease genetic studies. Gut 2001;49:773-776.

3. Henriksen M, Jahnsen J, Lygren I, et al. Change of diagnosis during the first five years after onset of inflammatory bowel disease: results of a prospective follow-up study (the IBSEN Study). Scand J Gastroenterol 2006;41:1037-1043.

4. Yantiss RK, Odze RD. Pitfalls in the interpretation of nonneoplastic mucosal biopsies in inflammatory bowel disease. Am J Gastroenterol 2007;102:890-904.

5. Nikolaus S, Schreiber S. Diagnostics of inflammatory bowel disease. Gastroenterology 2007;133:1670-1689.

6. Podolsky DK. Inflammatory bowel disease. N Engl J Med 1991;325:928-937.

7. Matsui T, Yao T, Sakurai T, et al. Clinical features and pattern of indeterminate colitis: Crohn's disease with ulcerative colitis-like clinical presentation. J Gastroenterol 2003;38:647-655. 
8. Geboes K, Colombel JF, Greenstein A, et al. Indeterminate colitis: a review of the concept -what's in a name? Inflamm Bowel Dis 2008;14:850-857.

9. Solberg IC, Vatn MH, Høie O, et al. Clinical course in Crohn's disease: results of a Norwegian population-based ten-year follow-up study. Clin Gastroenterol Hepatol 2007;5:1430-1438.

10. Solberg IC, Lygren I, Jahnsen J, et al. Clinical course during the first 10 years of ulcerative colitis: results from a populationbased inception cohort (IBSEN Study). Scand J Gastroenterol 2009;44:431-440.

11. Yang SK, Hong WS, Min YI, et al. Incidence and prevalence of ulcerative colitis in the Songpa-Kangdong District, Seoul, Korea, 1986-1997. J Gastroenterol Hepatol 2000;15:1037-1042.

12. Yang SK, Yun S, Kim JH, et al. Epidemiology of inflammatory bowel disease in the Songpa-Kangdong district, Seoul, Korea, 1986-2005: a KASID study. Inflamm Bowel Dis 2008;14:542549.

13. Park SH, Yang SK, Park SK, et al. Long-term prognosis of crohn's disease and its temporal change between 1981 and 2012: a hospital-based cohort study from Korea. Inflamm Bowel Dis 2014;20:488-494.

14. Lee HS, Park SH, Yang SK, et al. Long-term prognosis of ulcerative colitis and its temporal change between 1977 and 2013: a hospital-based cohort study from Korea. J Crohns Colitis 2015;9:147-155.

15. Lennard-Jones JE. Classification of inflammatory bowel disease. Scand J Gastroenterol Suppl 1989;170:2-6.

16. Park SH, Kim YM, Yang SK, et al. Clinical features and natural history of ulcerative colitis in Korea. Inflamm Bowel Dis 2007;13:278-283.

17. Ye BD, Yang SK, Cho YK, et al. Clinical features and long-term prognosis of Crohn's disease in Korea. Scand J Gastroenterol 2010;45:1178-1185.

18. Choi CH, Jung SA, Lee BI, et al. Diagnostic guideline of ulcerative colitis. Korean J Gastroenterol 2009;53:145-160.

19. Ye BD, Jang BI, Jeen YT, et al. Diagnostic guideline of Crohn's disease. Korean J Gastroenterol 2009;53:161-176.

20. Louis E. When it is not inflammatory bowel disease: differential diagnosis. Curr Opin Gastroenterol 2015;31:283-289.

21. Schumacher G, Sandstedt B, Kollberg B. A prospective study of first attacks of inflammatory bowel disease and infectious colitis. Clinical findings and early diagnosis. Scand J Gastroenterol 1994;29:265-274.

22. Lee YJ, Yang SK, Byeon JS, et al. Analysis of colonoscopic findings in the differential diagnosis between intestinal tuberculosis and Crohn's disease. Endoscopy 2006;38:592-597.
23. Ye BD, Yang SK, Kim D, et al. Diagnostic sensitivity of culture and drug resistance patterns in Korean patients with intestinal tuberculosis. Int J Tuberc Lung Dis 2012;16:799-804.

24. Ko JK, Lee HL, Kim JO, et al. Visceral fat as a useful parameter in the differential diagnosis of Crohn's disease and intestinal tuberculosis. Intest Res 2014;12:42-47.

25. Weng MT, Wei SC, Lin CC, et al. Seminar report from the 2014 Taiwan Society of Inflammatory Bowel Disease (TSIBD) spring forum (May 24th, 2014): Crohn's disease versus intestinal tuberculosis infection. Intest Res 2015;13:6-10.

26. Gan HT, Chen YQ, Ouyang Q, Bu H, Yang XY. Differentiation between intestinal tuberculosis and Crohn's disease in endoscopic biopsy specimens by polymerase chain reaction. Am J Gastroenterol 2002;97:1446-1451.

27. Epstein D, Watermeyer G, Kirsch R. Review article: the diagnosis and management of Crohn's disease in populations with high-risk rates for tuberculosis. Aliment Pharmacol Ther 2007;25:1373-1388.

28. Almadi MA, Ghosh S, Aljebreen AM. Differentiating intestinal tuberculosis from Crohn's disease: a diagnostic challenge. Am J Gastroenterol 2009;104:1003-1012.

29. Makharia GK, Srivastava S, Das P, et al. Clinical, endoscopic, and histological differentiations between Crohn's disease and intestinal tuberculosis. Am J Gastroenterol 2010;105:642-651.

30. Thia KT, Loftus EV, Jr., Sandborn WJ, Yang SK. An update on the epidemiology of inflammatory bowel disease in Asia. Am J Gastroenterol 2008;103:3167-3182.

31. Loke YK. Use of databases for clinical research. Arch Dis Child 2014;99:587-589.

32. Molodecky NA, Panaccione R, Ghosh S, Barkema HW, Kaplan GG; Alberta Inflammatory Bowel Disease Consortium. Challenges associated with identifying the environmental determinants of the inflammatory bowel diseases. Inflamm Bowel Dis 2011;17:1792-1799.

33. Kaplan GG. Pitfalls and perils of using administrative databases to evaluate the incidence of inflammatory bowel disease overtime. Inflamm Bowel Dis 2014;20:1777-1779.

34. Ngeow J, Eng C. New genetic and genomic approaches after the genome-wide association study era-Back to the future. Gastroenterology 2015;149:1138-1141.

35. Lee KM, Kim YS, Seo GS, Kim TO, Yang SK, IBD Study Group of the Korean Association for the Study of Intestinal Diseases. Use of thiopurines in inflammatory bowel disease: a consensus statement by the Korean Association for the Study of Intestinal Diseases (KASID). Intest Res 2015;13:193-207. 